\title{
Post-Lumbar Surgery Complex Regional Pain Syndrome
}

\author{
David E. Fish, MD
}

Background: This case report identifies a patient with complex regional pain syndrome Type 2 (causalgia) with sympathetically maintained pain in a distal extremity associated with an anterior sacroiliac fusion with local bone graft.

Case Description: A 65-year-old man underwent a sacroiliac fusion for chronic back pain that was temporarily relieved with percutaneous injections to the joint space and lower lumbar facets. The successful surgery with allograft had no intraoperative complications. The patient remained in the hospital post-operatively for four days with no complications. Four to six weeks post-op- erative, the patient began to have swelling and pain of the ipsilateral foot. The pain progressed with evidence of allodynia, hyperesthesia, and dystrophic changes in the foot. EMG and a nerve conduction study showed severe denervation that involved not only the muscles below the knee but the quadriceps as well, with ongoing irritability of a number of muscles, including those innervated by the femoral, sciatic, and gluteal nerves in the left leg. A diagnosis of complex regional pain syndrome Type 2 (causalgia) was made with the aid of a bone scan. A series of two diagnostic percutaneous chemical sympathectomies were undertaken, and the pain was sig- nificantly relieved. This suggested complex regional pain syndrome Type 2 with sympathetically maintained pain. The patient underwent aggressive physical therapy during rehabilitation; subsequent resolution of the pain syndrome occurred.

Conclusion: Complex regional pain syndrome Type 2 with sympathetically maintained pain is a condition that can result in serious disability and can be associated with spinal procedures and sacroiliac arthrodesis. Early intervention is recommended to provide long-term resolution of the condition.

Keywords: Sacroiliac, low back pain, CRPS, rehabilitation
Complex regional pain syndrome (CRPS) Types 1 and 2, known previously as reflex sympathetic dystrophy (RSD) and causalgia, respectively, depict a regional pain syndrome that may be characterized by intense burning and excruciating pain, allodynia, hyperalgesia, motor impairment, and autonomic and tissue changes in the region of the pain including edema, changes in skin blood flow, and/or abnormal pseudomotor activity (1). Both CRPS Types 1 and 2 share the same signs and symptoms, and CRPS Type 2 is differentiated from Type 1 only by the existence of a definable nerve lesion. These syndromes are further subdivided into sympathetically maintained

From: Department of Orthopaedics, David Geffen School of Medicine At UCLA, Los Angeles, CA Address Correspondence:

David E. Fish, MD, MPH

$124516^{\text {th }}$ Street Tower Bld $7^{\text {th }}$ Floor, Rm 715

Santa Monica, CA 90404

E-mail: dfish@mednet.ucla.edu

Disclaimer: There was no external funding in prepa-

ration of this manuscript.

Conflict of Interest: None

Manuscript received on 5/15/2005

Revision submitted on 6/2/2005

Accepted for publication on 6/3/2005 pain (SMP) and sympathetically independent pain (SIP), depending on the pain relief provided by sympatholytic therapies (2).

While the signs and symptoms of CRPS were recognized as early as 1864 in American Civil War soldiers, the underlying pathophysiological mechanisms have yet to be defined. Although an abundance of theories exist, many are unproven and remain controversial, and it is generally thought that multiple factors may play a role in the etiology of CRPS (3). Current leading views theorize that a pathological interaction between the somatic and sympathetic branch of the autonomic nervous system enables a sympathetic discharge that directly stimulates sensory nerves, thus producing the symptoms associated with CRPS. Specifically, sensory endings are thought to develop sensitivity to catecholamines and sympathetic efferent nerves by expressing new alpha-2 receptors, constituting an abnormal synapse, termed an ephapse, between sympathetic and somatic nerves (4).

CPRS has been associated with amyotrophic lateral sclerosis, and the condition has been diagnosed as a complication following a number of interventions, in- cluding venipuncture, arteriovenous graft replacement, thromboendarterectomy, breast reduction surgery, and work-related electrical injury (1,5-11). CRPS Type 2 development in the foot has been reported after automated laser diskectomy, suspected to be due to nerve injury to the L5 spinal root or spinal nerve (12). Review of the literature in PubMed did not reveal any reported cases of CRPS development following sacroiliac fusion surgery.

Sacroiliac fusion surgery may be performed on patients with intractable back pain that does not respond to conservative treatments, with the notion that stabilizing the joint may aid in providing pain relief (13). Spinal surgery is associated with a number of complications, among which include anesthesia complications, thrombophlebitis, deep vein thrombosis, neurological complications, infection, hardware fracture, and implant migration. We present a unique case of CRPS following anterior sacroiliac arthrodesis with local bone graft surgery, an unusual complication.

\section{Case Description}

The patient, a 65 -year-old male with a longstanding history of episodic low- 
er back pain secondary to degenerative changes of the lumbar spine, was admitted for an elective sacroiliac fusion. Past medical history revealed that in 1989 he had a lumbar laminectomy for a radiculopathy with good clinical results until April of 2003 when he developed pain in the left buttock localized in the sacroiliac region. His pain was brought on by walking and resolved only with rest. Following clinical evaluation, it was determined that the patient had both recurrent lumbar stenosis as well as sacroiliac joint instability, with the radiographic examination revealing degenerative changes at the left sacroiliac joint. The patient's pain progressively worsened such that he became limited in his functional ability to get dressed and to ambulate secondary to pain. The patient underwent several local SI joint injections in his left side, which provided temporary relief. After the facet injection showed some improvement, he then had radiofrequency of the L4-S3 medial branch nerves of the facet and sacroiliac joints on the left side.

Since all other conservative treatments and interventional procedures had failed to provide adequate pain relief, the patient opted for a left-sided sacroiliac fusion with local bone graft. Specifically, an iliac crest bone graft was taken from the internal aspect of the iliac wing at the level of the abductor tubercle, which was then impacted using a small punch to impact solid cancellous bone into the central portion of the SI joint throughout its inferior and superior directions. A special unicortical cancellous bone graft about $1 \mathrm{~cm}$ by $2 \mathrm{~cm}$ was then fit into a groove cut in the anterior-inferior aspect of the sacroiliac joint and extending less than $1 \mathrm{~cm}$ medially across the SI surface to $2 \mathrm{~cm}$ laterally. This was then placed as an onlay solid bone graft across the SI joint, after which a five-hole pelvic reconstruction plate was secured with one screw into the sacrum and two screws in the iliac wing at the inferior level anteriorly. A second plate was then placed across the sacroiliac joint with one screw into the sacrum and two screws in the iliac wing of the superior lateral aspect of the joint. Further bone graft debris was then placed in the central portion between these two plates to complete the fusion; drains were placed. The successful surgery with autograft had no intraoperative complications. Fig. 1 demonstrates the sacroiliac fusion.

The patient tolerated the surgical intervention well and remained in the hospital four days with no post-operative complications. By post-operative day four, the patient was ambulating well with crutches without additional assistance. However, four to six weeks post-operative, the patient developed swelling, dark discoloration, and pain in his left foot that radiated from his back at the site of the surgery (Fig. 2). He also developed numbness in the lateral and then medial aspect of his left foot and in his toes. The pain progressed, with evidence of allodynia, hyperesthesia, and reflex dystrophic changes in the foot. The pain was worse in the evening, and there were no bowel or bladder symptoms.

Approximately three weeks later the patient was sent to a neurologist to confirm the possibility of a nerve compro- mise during the procedure. Sensation was intact to pinprick in all four extremities with allodynia in the region of his left leg innervated by the sciatic nerve, and there was decreased weight bearing in the left leg. Manual muscle testing was 5/5 in all four extremities apart from eversion, toe extensor, and dorsiflexion on the left side, which were $3 / 5$ due mainly to pain and not true weakness.

An EMG and a nerve conduction study showed severe denervation involving not only the muscles below the knee but the quadriceps as well. There was also had ongoing irritability of a number of muscles, including those innervated by the femoral, sciatic, and gluteal nerves in the left leg. Chronic denervation changes were noted in most of the left lower extremity. The patient was sent to a comprehensive spine center for further evaluation. On presentation, the patient had classic symptoms of CRPS, which included skin mottling, swelling, allodynia, erythema, and hyperpathia.

The patient underwent a three-phase bone scan, which demonstrated diffusely increased blood flow and blood pool activity to the left foot with delayed concentration of tracer within the periarticular regions of the tarsal, tarsometatarsal, and metatarsophalangeal joints, confirming the diagnosis of CRPS (Fig. 3).

The bone scan also revealed a severe arthritic reaction along the left posterolateral margin of L2 through L4, and a moderate arthritic reaction in the bilateral sacroiliac joints and to a lesser degree along the right lateral margin of the entire lumbar spine. A series of two diagnostic per-

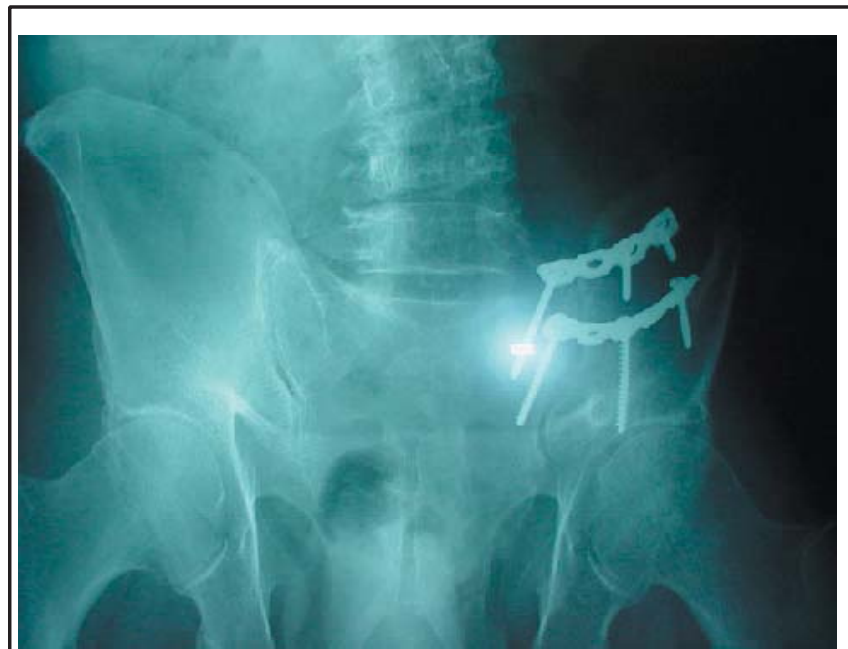

Fig. 1. X-ray AP pelvis showing sacroiliac joint fusion

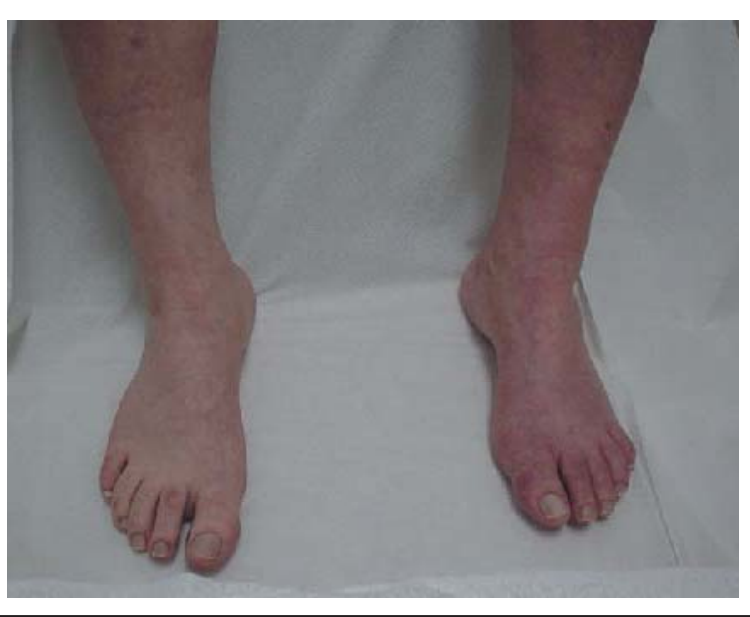

Fig. 2. Bilateral feet showing erythema and swelling in the left lower extremity 


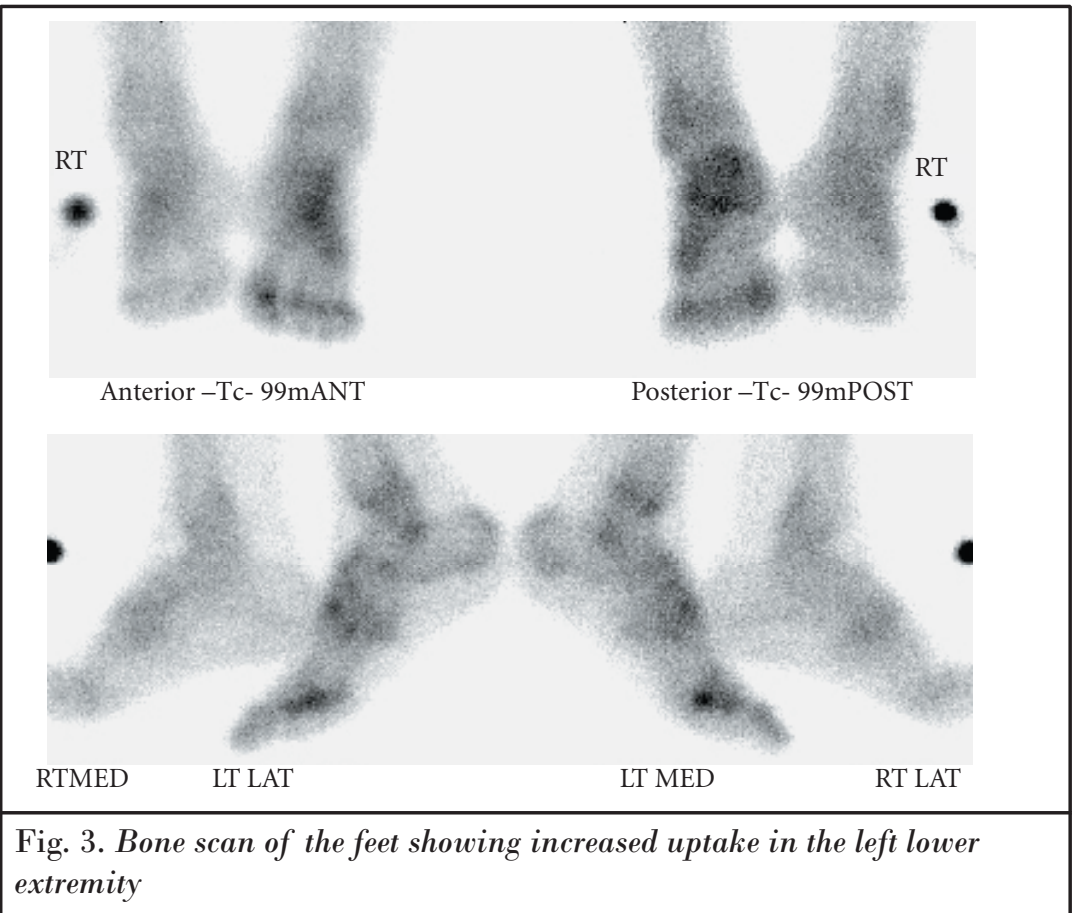

cutaneous chemical sympathectomies at L2 and L3 provided significant pain relief, suggestive of CRPS Type 2 with sympathetically maintained pain. The patient subsequently had three sympathetic blocks, anti-seizure medication, and aggressive physical therapy. The patient had dramatic improvement after treatment and no recurrence of the CRPS developed.

\section{DISCUSSION}

This case illustrates an incidence of CRPS with sympathetically maintained pain following anterior sacroiliac fusion with local bone graft surgery. Prior to a definitive diagnosis, this patient showed signs consistent with CRPS Type 2, including continued pain, allodynia, hyperesthesia, and dystrophic changes in the foot.

CRPS often affects the hands or feet, as with this particular case, but it may also involve and even spread to the entire extremity. While the etiology of CPRS may typically be an inciting injury such as a sprain, dislocation, fracture, contusion, or crush injury, other causes may be spontaneous or unknown $(14,15)$. Moreover, there exists no correlation between the severity of an injury and the resultant intensity of a patient's symptoms (15). The pain is distinct in that it is disproportionately exaggerated with regard to the incit- ing injury, tends to begin days to weeks following the injury, persists for longer period of time than is expected for an injury to heal, and does not remain isolated within a dermatome.

While the precise pathogenesis of this syndrome remains undefined, we postulate that the mechanism for the development of CRPS Type 2 in this specific instance may have been due to a peripheral nerve compromise during sacroiliac arthrodesis surgery. CPRS complications including nerve compromise are more commonly seen in the periphery. While other central nervous system factors may have been involved in the pathophysiology of the disease, the presence of distal CRPS without an injury to the ankle implies that a proximal injury of a peripheral nerve may have occurred, which then led to the manifestations of CRPS.

A similar case reported in the literature describes the development CRPS Type 2 with sympathetically maintained pain in the foot following suspected L5 spinal root or spinal nerve injury from L4-L5 automated laser diskectomy (12). Another case describes the development of CRPS distally in the hand of a hemodialysis patient following the placement of a polytetrafluoroethylene (PTFE) arteriovenous graft in his ipsilateral forearm (8). Thus, our case corroborates the notion that CRPS does not have to start at the site of trauma or surgery, but can result from a proximal nerve injury.

Current treatment guidelines for CRPS recommend an interdisciplinary approach aimed at attaining functional restoration, placing emphasis on pain management, rehabilitation, and psychological therapy (9).

Mild to moderate sacroiliac joint pain can be managed conservatively with analgesics, anti-inflammatory drugs, and physical therapy. Severe sacroiliac joint pain can be incapacitating and more challenging to manage. Fluoroscopicallyguided intra-articular, local anestheticsteroid injections, followed by joint manipulation, can be effective. Intracapsular injections of glycerin, glucose, and phenol may also be beneficial in some patients.

The use of neuroaugmentation to manage pain of synovial origin has not been reported previously. Sacral nerve root stimulation in particular has been used to manage urinary bladder dysfunction and pain, but not sacroiliac joint pain.

\section{ConcLusion}

In summary, this is a case of CRPS Type 2 with sympathetically maintained pain, a condition that can result in serious disability, which can be associated with spinal procedures and sacroiliac arthrodesis. Thus, we believe that CRPS should be included as a potential differential diagnosis in patients suffering from distal limb pain following spine surgery. Spine surgeons and pain medicine physicians must be able to recognize CRPS and promptly refer patients for diagnosis and treatment, as early intervention is recommended to provide long-term resolution of the condition.

\section{AuthOR AfFilition:}

\section{David E. Fish, MD}

Associate Clinical Professor

Department of Orthopaedics

Physical Medicine and Rehabilitation

David Geffen School of Medicine at

UCLA

$124516^{\text {th }}$ Street Tower Bldg. $7^{\text {th }}$ Floor, Rm 715

Santa Monica, CA 90404

E-mail: dfish@mednet.ucla.edu 


\section{RefERENCES}

1. DeLee J, Drez D. Complex Regional Pain Syndromes Including Reflex Sympathetic Dystrophy and Causalgia. In DeLee and Drez's Orthopaedic Sports Medicine, 2nd ed., W.B. Saunders Company, 2003, pp 441-460.

2. Stanton-Hicks MD, Burton AW, Bruehl SP, Carr DB, Harden RN, Hassenbusch SJ, Lubenow TR, Oakley JC, Racz GB, Raj PP, Rauck RL, Rezai, AR. An updated interdisciplinary clinical pathway for CRPS: Report of an expert panel. Pain Practice 2002; 2: 1-16.

3. Stanton-Hicks M, Baron R, Boas R, Gordh $T$, Harden N, Hendler N, Koltzenburg M, Raj P, Wilder R. Complex regional pain syndromes: Guidelines for therapy. Clin J Pain 1998; 14:155-166.

4. Baillet G, Planchon CA, Thamgac F, Thomassin M, Foult JM. Complex regional pain syndrome after thromboendarterectomy. Clin Nucl Med 2002; 27:619-621.
5. Dontigny RL. Function and pathomechanics of the sacroiliac joint. A review. Phys Ther 1985; 65:35-44.

6. Kim CT, Bryant P. Complex regional pain syndrome (type I) after electrical injury: A case report of treatment with continuous epidural block. Arch Phys Med Rehabil 2001; 82:993-995.

7. Mori H, Watanabe M, Akahane M, Yajima C, Ohtomo K. A case of venipuncture-induced complex regional pain syndrome. Nippon Igaku Hoshasen Gakkai Zasshi 2002; 62:834-835.

8. Pandita D, Danielson BD, Potti Anil, Lo TS, Buettner A. CRPS type-1: A rare complication of arteriovenous graft placement. J Rheumatol 1999; 26:2254-2256.

9. Shibata M, Abe Km Jimbo A, Shimizu T, Mihara M, Sadahiro S, Yoshikawa H, Mashimo T. Complex regional pain syndrome type I associated with amyotrophic lateral sclerosis. Clin J Pain 2003; 19:69-70.

10. Sibanc B, Lesnicar G. Complex regional pain syndrome and lyme borreliosis: Two different diseases? Infection 2002; 30: 396-399.

11. Papay FA, Verghese A, Stanton-Hicks M, Zins J. Complex regional pain syndrome of the breast in a patient after breast reduction. Ann Plast Surg 1997; 39:347-352.

12. Plancarte R, Calvillo O. Complex regional pain syndrome type 2 (causalgia) after automated laser discectomy. A case report. Spine 1997; 22:459-462.

13. Canale ST, Campbell WC. "Arthrodesis." Campbell's Operative Orthopaedics, $10^{\text {th }}$ ed. St Louis, MO: Mosby, 2003 ; Chpt 13: 597-610

14. Baron R, Levine JD, Fields HL: Causalgia and reflex sympathetic dystrophy: Does the sympathetic nervous system contribute to the generation of pain? Muscle Nerve 1999; 22:678-695.

15. Pittman DM, Belgrade MJ. Complex regional pain syndrome. Am Fam Physician 1997; 56.2265-2270, 2275-2276. 